\title{
tentas \\ Depuis 30 ans, aujourd'hui et demain : transmettre la science biomédicale en langue française \\ $+1$
}

> En 2015, quelle passerelle du savoir est médecine/sciences et comment doit évoluer cette passerelle ? Telles seront les questions centrales du colloque anniversaire des 30 ans de la revue, qui se tiendra le 12 mars à l'auditorium des biotechnologies de l'Institut Pasteur à Paris. Aujourd'hui, comme il y a un ou trente ans « [...] la science ne se limitait pas à un exposé des résultats. Une structuration de ces résultats autour de concepts et d'un regard critique, en bref une pensée de ces résultats, s'avérait et s'avère toujours nécessaire et la langue maternelle est un support essentiel de cette pensée. » [1].

- Nous poserons d'abord la question «Comment lit-on une revue scientifique aujourd'hui ? $\gg \mathrm{m} / \mathrm{s}$ a fait évoluer sa maquette et son format pour proposer au fur et à mesure de nouveaux modes de lecture. $\grave{A}$ l'origine, lorsque l'accès aux revues était difficile et l'acheminement postal lent, l'invention des Brèves permit une lecture panoramique rapide de l'actualité. $\mathrm{m} / \mathrm{s}$ fut imitée par nombre de prestigieuses revues anglo-saxonnes. Puis, le numérique et l'accès en ligne (Biblioinserm/ Bibliovie) rendirent les Brèves à leur tour trop tardives. Comment, en un temps où personne n'a plus le temps, pouvons-nous porter à la connaissance de nos lecteurs la profondeur du questionnement scientifique d'une recherche et non seulement quelques résultats récents? Prenons comme exemple l'épidémie d'Ebola sans précédent frappant l'Afrique de l'Ouest. Au 29 décembre 2014, le nombre de morts s'élevait, selon l'Organisation mondiale de la santé (OMS), à 7842 sur un total de 20081 cas enregistrés dans les trois pays les plus touchés, la Sierra Leone, le Liberia et la Guinée où l'épidémie a éclaté il y a un an. Dès le numéro de juin-juillet 2014 [2], $\mathrm{m} / \mathrm{s}$ rendait compte de l'épidémie, en s'appuyant sur les analyses les plus récentes d'auteurs dont nous avions publié les travaux sur Ebola dès 2002 [3]. Mais Ebola n'est pas que le nom d'un virus et l'épidémie va " générer d'importantes conséquences économiques, politiques et sociales », comme le souligne Antoine Flahaut dans son Éditorial d'octobre 2014 [4]. Comment éclairer notre lecteur sur les multiples facettes des sujets que nous publions, alors que souvent la clarté d'un article vient de sa focalisation sur un aspect particulier du problème ? Comment permettre la synthèse à un instant donné de la connaissance ? Le « web sémantique » ouvre certainement des pistes à explorer, et il faudra développer de nouvelles offres permettant au lecteur d'interagir en temps réel avec le contenu d'un article pour bénéficier effectivement de la richesse des contenus de la revue. Comment redonnons-nous du temps au temps et une profondeur à l'actualité ? La première manière est évidemment d'analyser pas à pas les avancées d'un domaine et ne pas s'arrêter à une annonce spectaculaire.
C'est ce qui nous a permis d'annoncer - avec circonspection - la découverte des STAP, ces cellules reprogrammées en cellules souches par la magie d'un choc acide et, sans surprise, d'avertir nos lecteurs de la rétractation de l'article [5]. Une autre manière de « donner à penser » est d'éclairer le sens des concepts que nous utilisons et nous pouvons donner en exemple récent le beau texte d'Hervé Watier sur les définitions croisées et comparées de la biothérapie et des thérapies ciblées [6].

- Lors de la seconde session du colloque, nous regarderons $\mathrm{m} / \mathrm{s}$ face aux nouvelles technologies du vivant : comment une revue rend-elle compte des avancées technologiques si essentielles aux découvertes dans nos disciplines? De plus en plus régulièrement, nous publions des dossiers techniques permettant à nos lecteurs de se familiariser avec les différentes offres de technologies émergentes comme l'editing du génome, la métagénomique, la protéomique quantitative, la PCR digitale et son application à la détection d'ADN tumoral circulant (dans le dernier numéro [7]), ou encore l'optogénétique dans le prochain, ou des séries comme la série chémobiologie. $\varepsilon t$, comme toujours pour $\mathrm{m} / \mathrm{s}$, rendre compte n'est pas simplement exposer le progrès dans une naïveté admirative, mais éclairer aussi son revers. Par exemple, l'une des questions d'économie de la santé de 2014 aura été le coût du sofosbuvir (Sovaldi, Gilead), un antiviral indiqué dans le traitement des hépatites C [8], permettant un taux de guérison de $90 \%$ au lieu des $50 \%$ avec les traitements précédents. Initialement fixé à 57000 euros pour les trois mois de traitement, le prix a été renégocié en novembre 2014 à 41000 euros. C'est un coût qui reste énorme, puisque le traitement pourrait s'adresser à plus de 60000 personnes actuellement rien qu'en France où la population infectée est estimée à 200000 personnes. Comme le dit l'adage, «la santé n'a pas de prix, mais elle a un coût », comment rendre ce coût supportable à nos systèmes d'assurance santé sans porter atteinte à la solidarité ou à d'autres actions nécessaires comme la prévention ? L'économie de la santé, déjà présente dans nos colonnes $[9,10]$, le sera en conséquence de plus en plus dans les prochaines années.

- C'est autour des questions de la complexité que s'articulera notre troisième table ronde. Nous aurons ici à nous confronter à l'un des changements conceptuels les plus délicats à gérer, surtout en termes de publication scientifique, le passage du réductionnisme/ déterminisme à la biologie des systèmes et son corrélat statistique. Depuis un peu plus d'un siècle, et avec un succès évident, déterminer 
c'est réduire la maladie à ses symptômes et réduire les symptômes à leur cause. C'est la démarche de la médecine scientifique, la médecine basée sur les preuves, définie par Claude Bernard dans son «Introduction à la médecine expérimentale » : «(il) exercera son influence sur les maladies dès qu'il en connaitra expérimentalement le déterminisme exact, c'est-à-dire la cause prochaine » [11]. Pour le scientifique, déterminer c'est d'abord assigner des limites, ou encore spécifier ce qui distingue un objet d'un autre et l'assigner à une catégorie. Mais, plus couramment, déterminer ce sera rechercher les mécanismes, leurs origines, leurs moteurs dynamiques, qui produisent un effet. Décrire cette chaîne ordonnée dans laquelle chaque élément dépend d'un certain nombre d'autres d'une façon telle qu'il peut être prévu, c'est l'objet même de la recherche scientifique, et pour reprendre Claude Bernard: «la critique expérimentale met tout en doute, excepté le principe du déterminisme scientifique. » [12]. Comment ce déterminisme si fécond pour la médecine basée sur les preuves s'articule-t-il aujourd'hui avec la variabilité statistique des événements à l'échelle moléculaire et leur intégration dans la biologie des systèmes? Pour poursuivre cette réflexion, nous vous renvoyons à la remarquable série de textes sur «Penser les rapports entre hasard, réductionnisme et holisme dans le cancer » dans notre numéro de juin-juillet 2014 [13]. Dès 2009, $\mathrm{m} / \mathrm{s}$ publiait un premier numéro thématique dédié à la biologie des systèmes, complété en janvier 2010. Une autre approche de cette complexité est la biologie de synthèse et, ici encore, $\mathrm{m} / \mathrm{s}$ en a rendu compte très tôt, par exemple en vous tenant informé des résultats de la compétition internationale iGEM ${ }^{1}$ dès 2007 [14]. La biologie de synthèse a fait en 2014 quelques pas de plus avec l'expression d'un chromosome synthétique dans une levure et une lignée de $\varepsilon$. coli dont l'ADN intégrait deux nouvelles lettres artificielles. Le revers de la médaille, c'est aussi de pouvoir produire des « Frankenvirus », comme cette reconstitution du virus de la grippe espagnole à partir de fragments viraux aviaires circulant chez des canards [15, 16].

- La quatrième et dernière session aura la lourde tâche d'ouvrir des perspectives vers notre avenir : comment publiera-t-on demain dans $\mathrm{m} / \mathrm{s}$ ? Nous pouvons évidemment nous appuyer sur une longue expérience de formats variés jusqu'à cette synthèse si élégante de la science et d'une réflexion sociologique que nous offre Bertrand Jordan chaque mois dans ses Chroniques génomiques. Nos invités à ce colloque nous guideront vers de nouveaux chemins de la connaissance et de la formation universitaire et postuniversitaire. Nous nous interrogerons par exemple sur l'articulation entre les articles publiés dans $\mathrm{m} / \mathrm{s}$ (parlera-t-on d'ailleurs encore de numéros ou d'articles dans 5 ans ?) et les nouvelles pratiques de formation permises par le numérique comme les MOOC (cours numériques ouverts massifs) ou les Smart games (jeux élégants) ? Les articles et schémas de la revue sont de fréquents supports des cours actuels. Peuvent-ils être demain la base d'un nouvel enseignement numérique interactif ? Comment développer de tels programmes et comment en valider l'efficience ? Comment permettre à d'autres scientifiques également de s'exprimer dans leur langue natale. Nous gardons en projet la possibilité de traduire en chinois nos textes et pourquoi pas en espagnol, en portugais... car, comme l'exprimait récemment Anne-Marie Moulin, «Si l'échec d'une seule langue à incarner la perfection d'un langage scientifique est patent, une question demeure, à notre époque

${ }^{1}$ GGEM : international genetically engineered machine competition. d'éloge de la diversité du vivant, celle des bénéfices scientifiques et extrascientifiques de la diversité des langues » [17]. Toujours plus d'offres, me direz-vous, mais avec une interrogation constante : qui nous lit aujourd'hui et qui nous lira demain?

Ce colloque, grâce à vous chers auteurs et chers lecteurs, est un nouveau départ. Car 2015 nous réserve bien des émerveillements, des premiers pas de la thérapie cellulaire utilisant des cellules souches embryonnaires - comme vient de le faire l'équipe de Philippe Ménasché à Paris pour le cœur - ou des cellules souches reprogrammées (iPS) dans l'œil pour l'équipe de Masayo Takahashi à Kobe, jusqu'à l'essor des grands programmes de modélisation du cerveau, l'European Human Brain Project ou l'Américain Brain. 30 ans déjà, 30 ans seulement. À votre tour d'entrer dans la danse...

Since 30 years and tomorrow too: publishing biomedical science in French

\section{LIENS D'INTÉRÊT}

L'auteur déclare n'avoir aucun lien d'intérêt concernant les données publiées dans cet article.

Hervé Chneiweiss
Rédacteur en chef de médecine/sciences
équipe plasticité gliale et tumeurs cérébrales
Neuroscience Paris Seine-IBPS
UMR8246 CNRS/U1130 Inserm/UMCR18
Université Pierre et Marie Curie
7 , quai Saint Bernard
75005 Paris, France
herve.chneiweiss@inserm.fr

\section{RÉFÉRENCES}

1. Chneiweiss H. À quoi sert donc une revue scientifique en langue française ? Med Sci (Paris) $2014 ; 30: 7-8$.

2. Reynard 0 , Volchkov V, Peyrefitte $C$. Une première épidémie de fièvre à virus $\varepsilon$ bola en Afrique de l'Ouest. Med Sci (Paris) 2014 ; $30: 671-3$.

3. Baize $S$, Deubel V. Identification d'un co-facteur pour l'entrée des filovirus Ebola et Marburg. Med Sci (Paris) $2002 ; 18: 13-14$

4. Flahault A. Les trois paradoxes du risque épidémique. Med Sci (Paris) 2014 ; 30 : 823-4.

5. Coulombel L. Rétractation - Après les iPS, voici les STAP, une nouvelle méthode de reprogrammation en cellules souches pluripotentes Med Sci (Paris) 2014 ; 30 : 214-7.

6. Watier H. Biothérapies, immunothérapies, thérapies ciblées, biomédicaments... Med Sci (Paris) $2014 ; 30: 567-75$.

7. Perez-Toralla K, Pekin D, Bartolo JF, et al. PCR digitale en microcompartiments : détection sensible de séquences d'acides nucléiques rares. Med Sci (Paris) $2015 ; 31: 84$-92.

8. Pol S. Virus de l'hépatite C. Med Sci (Paris) 2013 ; 29 : 998-1003.

9. Detournay B. L'évaluation économique dans le champ de la santé. Med Sci (Paris) 2014 ; 30 : 584-8.

10. Paris V. L'impact de la crise économique sur les systèmes de santé des pays de l'OCDE. Med Sci (Paris) $2014 ; 30: 910-5$.

11. Bernard C. Introduction à la médecine expérimentale. Paris : Champs Flammarion, $1984: 298$.

12. Bernard C. Introduction à la médecine expérimentale. Paris : Champs Flammarion, $1984: 244$.

13. Morange M. Les modèles explicatifs du cancer. Med Sci (Paris) 2014 ; $30: 679-82$.

14. Peccoud J, Coulombel L. Une competition de biologie synthétique Med Sci (Paris) 2007 ; 23 : $551-2$.

15. Lina B. Potentiel pandémique des virus influenza aviaires circulant actuellement et ayant des caractéristiques proches du virus de 1918. Med Sci (Paris) $2014 ; 30: 851-3$.

16. Mouret S. Un monde grippé (Frédéric Keck). Med Sci (Paris) $2013 ; 29: 94-7$.

17. Moulin AM. La guerre des langues n'aura pas lieu. Med Sci (Paris) 2014 ; 30 : 479-80.
TIRÉS À PART

H. Chneiweiss 